\title{
Editorial: Intestinal Dysbiosis in Inflammatory Diseases
}

\author{
Gislane Lelis Vilela de Oliveira ${ }^{1,2 *}$, Cristina Ribeiro de Barros Cardoso ${ }^{3}$, Veena Taneja ${ }^{4}$ \\ and Alessio Fasano ${ }^{5,6,7}$ \\ ${ }_{1}^{1}$ Microbiology Program, Institute of Biosciences, Humanities and Exact Sciences, São Paulo State University, \\ Sao Jose do Rio Preto, Brazil, 2 Department of Food Engineering and Technology, Institute of Biosciences, Humanities and \\ Exact Sciences, São Paulo State University, Sao Jose do Rio Preto, Brazil, ${ }^{3}$ Department of Clinical Analysis, Toxicology and \\ Food Sciences, School of Pharmaceutical Sciences of Ribeirão Preto, University of São Paulo, Ribeirão Preto, Brazil, \\ ${ }^{4}$ Department of Immunology and Department of Medicine, Division of Rheumatology, Mayo Clinic Rochester, Rochester, \\ MN, United States, ${ }^{5}$ Division of Pediatric Gastroenterology and Nutrition, Center for Celiac Research, Mucosal Immunology \\ and Biology Research Center, Massachusetts General Hospital, Boston, MA, United States, 6 Gastroenterology and Nutrition, \\ Harvard Medical School, Boston, MA, United States, ${ }^{7}$ European Biomedical Research Institute of Salerno, Salerno, Italy
}

Keywords: inflammatory diseases, gut microbiota, dysbiosis, gut permeability, microbiota modulators

\section{Editorial on Research Topic}

\section{Intestinal Dysbiosis in Inflammatory Diseases}

The human body is densely colonized by more than a trillion of commensal and mutualistic microorganisms, including archaea, bacteria, virus, bacteriophages and fungi (1-3). The majority of these microbial communities, referred to as the microbiota, resides within the intestine and is influenced by several factors that shape the establishment of a predominant protective, diverse or potentially harmful population in this ecosystem. As an example, the mode of delivery exposes the neonate to different microorganisms depending on the birth route. Vaginal-delivered babies are usually colonized by a more protective microbiota, while C-section newborns first contact common skin bacteria. Similarly, breastfeeding is mostly advantageous because of the immune protection mediated by antibodies, lactoferrin and anti-microbial peptides present in human milk, besides the known gut protective microorganisms Bifidobacterium and Lactobacillus that usually are not present in infant formulas. These factors may protect the newborn to infections, allergies and other future morbidities. In addition, by the introduction of solid foods and diet modifications throughout the infancy, this early colonization may shifts towards and adult-like microbiome, that may also be susceptible to changes by the use of medications such as antibiotics. Indeed, these drugs can alter the local ecosystem and disrupt the neonatal microbiome that could also be shaped by host genetics (4). Therefore, a variety of conditions can alter the human microbiota and its unbalance can potentially predispose the individuals to inflammatory diseases.

Usually, the intestinal microbiota plays an important role in a variety of physiological processes such as the maturation of host immunity, food fermentation and digestion, vitamin synthesis, regulation of intestinal hormones or host and drugs' metabolism. These functions exerted by the gut microorganisms have in common the capacity to impact the inflammation and the immune response that are triggered in chronic diseases $(5,6)$. In fact, the microbial-mucosal immune system interactions are tightly regulated, mainly contributing to homeostasis and eubiosis conditions in the gastrointestinal tract $(1,2)$. This tolerogenic response in the gut is fundamental to avoid excessive 
reactions to the local commensal microbiota (or to food antigens) meanwhile maintains the immunological competence in a stand by condition, able to prompt react if a pathogenic invasion occurs. However, a combination of environmental factors such as a gastrointestinal infection and genetic susceptibility can lead to a tolerance breakdown and intestinal dysbiosis, with either decrease or expansion of certain commensals, reduced diversity with an increase in pathobionts, resulting in altered function of this ecosystem (7-9). The outcomes of these deregulated interactions at the intestinal mucosa can have systemic effects in host immunity and contribute to the development of the chronic inflammatory or autoimmune diseases, as outlined above $(10,11)$.

This Research Topic of Frontiers in Immunology, Mucosal Immunity section, included reviews, mini-reviews, hypothesis and theory, besides original research articles describing microbiome/metabolomics studies in mice and humans. These works evaluated the intestinal dysbiosis in inflammatory diseases as well as the crosstalk between the commensal microbes and the immune system. Furthermore, the role of the microbiota modulators (diet, pre-/probiotics, and fecal microbiota transplantation) have been discussed, focusing in the reestablishment of the gut immune homeostasis in chronic inflammatory conditions. A total of 24 articles accepted in this Research Topic involved contributions from 178 researchers and the main findings are briefly discussed in this editorial.

Considering the numerous molecules found in the gut ecosystem, quorum sensing represents small signaling molecules produced by bacteria, depending on the density of these microorganisms, with a subsequent regulation of their gene expression and bacterial physiology (12). These products are detected by microbes, interact with the host cells and contribute to gastrointestinal homeostasis (13). Coquant et al. showed the importance of the quorum sensing molecules, focusing on type I autoinducers, such as $\mathrm{N}$-acyl-homoserine lactones (AHL). The authors pointed to the impact of these molecules on the innate and adaptive immune system, with important anti-inflammatory effects in experiments involving intestinal cell lines, thus indicating a perspective for the future use of natural or synthetic AHL to regulate gut inflammation and inflammatory bowel diseases (IBD).

Intestinal microbes may also produce short-chain fatty acids (SCFAs), which are end products from the fermentation of ingested dietary fibers. These molecules can bind specific receptors on mucosal cells and impact the local or systemic immunity, by inducing regulatory $\mathrm{T}$ cells (Tregs) and an antiinflammatory response that control the exacerbated reactions in the gut mucosa, thus contributing to the maintenance of the barrier integrity $(14,15)$. Moreover, epithelial or mucosal immune cells express pattern recognition receptors (Toll-like receptors - TLRs, NOD-like receptors - NLRs, and others) that also contribute to the sensing of the host microbiota and regulation of inflammatory processes (16). Interestingly, on this Research Topic, Elias-Oliveira et al. emphasized the interactions between NLRs and gut microbiota, showing the dual role of these molecules in promoting inflammation or protecting against the harmful effects of dysbiosis-driven diseases, such as IBD, type 1 or type 2 diabetes and obesity.

Then, to emphasize the importance of gut microbiota in inflammatory conditions, three original research articles explored this subject in animal models (17-28). Watanabe et al. evaluated the importance of these microorganisms in chronic kidney disease (CKD), by treating C57BL/6 mice, which were submitted to unilateral ureteral obstruction (UUO), with broad-spectrum antibiotics. The depletion of gut microbes induced a decrease in IL-1 $\beta$, IL-4, IL-6, GM-CSF, MIP$1 \beta$, fibronectin and type I collagen, improving the renal damage. Moreover, in Myd88 ${ }^{\Delta \mathrm{IEC}}$ mice, the bacterial translocation to mesenteric lymph nodes, after UUO, was lower than in WT mice. The reduced expression of inflammatory cytokines and chemokines in Myd88 ${ }^{\Delta \mathrm{IEC}}$ mice suggested that the role of the gut microbiota in CKD pathogenesis is related to the innate immune receptors' signaling. The second study, performed by Omura et al. evaluated the gut microbiota in mouse models of acute flaccid myelitis and multiple sclerosis (MS), which are inflammatory conditions that affect the central nervous system (CNS). To achieve this goal, researchers infected SJL/J mice with Theiler's murine encephalomyelitis virus (TMEV) and observed that although there were no significant differences in beta or alpha diversities between TMEV infected mice and controls, Dorea, Marvinbryantia, and Coprococcus genera changed after TMEV infection and correlated with immune molecules expression ( $\mathrm{T}$ cell receptor, Immunoglobulin genes, CD109) in spinal cords. Lastly, Seo et al. demonstrated that the administration of an agonistic antibody to TREM-1 (triggering receptor expressed on myeloid cell-1) led to an improvement in dysbiosis, restored epithelial barrier and ameliorated colitis in mice. Besides that, the beneficial effect of $\alpha$-TREM- 1 was dependent on CD177+ neutrophils and macrophages, via TLR4/Myd88 signaling.

Dysbiosis observed in autoimmunity is associated with impaired epithelial barrier function, systemic inflammation and decreased regulation in the gut mucosa (29-32), as a result of a variety of mechanisms stimulated or amplified by a dysbiotic microbiota $(31,33)$. In a review article from Brown et al. authors compiled data on intestinal dysbiosis in systemic lupus erythematosus, rheumatoid arthritis, and multiple sclerosis, discussing the contribution of the gut microbiota and SCFAs, with an additional special focus on recent studies linking the molecules from tryptophan metabolism to the protection or induction of autoimmune diseases. In addition, Hou et al. revised the intricate communication among leukocytes, intestinal stem cells (ISC) and gut microbiota, showing that this interaction is extremely important for the regenerative capacity of the gut epithelial cells. Indeed, signals derived from gut microbes affect the ISC development; i.e., while Tregs induce ISC renewal, the Th1/Th2/Th17 lymphocytes inhibit ISC activity. Besides that, authors discussed the importance of organoids derived from ISC to study this crucial interaction that could provide promising treatments for IBD.

Still on IBD, epidemiological studies showed higher incidence of IBD in patients with chronic obstructive pulmonary disease 
(COPD), and intestinal dysbiosis in patients with respiratory diseases, pointing to the importance of the gut-lung axis in health and disease (34-36). Two reviews explored this connection in the context of IBD vs. COPD and in COVID-19. Raftery et al. reviewed what is considered a "healthy microbiota" in the human gut and lungs. They also showed that intestinal dysbiosis (via bacteria metabolites) affects the lung homeostasis and may result in systemic effects on the immune system or production of inflammatory cytokines. De Oliveira et al. reviewed the gut-lung axis concepts and suggested that intestinal dysbiosis may affect the immune responses in the lungs and disease severity in COVID-19. Preliminary studies have shown the persistence of SARS-CoV-2 in the gastrointestinal system, and not only intestinal dysbiosis, but also nasopharyngeal and pulmonary dysbiosis in COVID-19 patients.

In a human cross-sectional study, Cortez et al. detected differences in the gut microbial composition between pediatric patients with primary sclerosing cholangitis (PSC) and those with PSC along with ulcerative colitis (UC). In patients under 10 years old, Streptococcus and Veillonella genera were increased in the PSC group when compared with controls, while Veillonella and Escherichia-Shigella were higher in the PSC+UC subjects. The increased Veillonella was associated with active disease, and this genus, in addition to Megasphaera, correlated with bilirubin and gamma-glutamyl transferase. These results suggested an association between disease activity and intestinal microbiome, thus opening new avenues for the diagnosis and prognosis of pediatric PSC. In another study, Xu et al. detected dysbiosis and increased intestinal permeability in patients with encephalitis, with predominance of the Proteobacteria phylum, and Parabacteroides and Oscillospira genera, besides increased plasma concentrations of D-lactate, intestinal fatty acid-binding protein, lipopolysaccharides, and lipopolysaccharide-binding protein. Furthermore, the levels of SCFAs (acetate, propionate and butyrate) were decreased in fecal samples from patients, while there was a direct correlation between the cerebrospinal fluid/serum albumin ratio and Ruminococcaceae relative abundance. Altogether, these data indicated that gut microbiota and their metabolites could play a role in brain injury and encephalitis' severity.

Regarding the applicability of microbiota modulators in inflammatory conditions, several original research articles investigated this question in experimental models. In autoimmune diseases, Kim et al. analyzed the effect of multistrain probiotics (IRT5) containing Lactobacillus acidophilus, L. casei, L. reuteri, Bifidobacterium bifidum, and Streptococcus thermophiles in non-obese diabetic (NOD) mice. Compared to controls, the IRT5 supplementation during 36 weeks decreased the diabetes incidence, pancreatic inflammation, insulin autoantibodies levels, intestinal barrier permeability, and increased the $\beta$-cell mass in NOD mice. Gusmao-Silva et al. administered probiotic bacteria (Lactococcus lactis NCDO 2118) carrying a recombinant protein (Hsp65) in chronic and acute mouse models of arthritis, and showed that this combination suppressed arthritis onset in both models and induced regulatory $\mathrm{CD}^{+} \mathrm{LAP}^{+} \mathrm{T}$ cells. In addition, the probiotic supplementation decreased IL-17, IFN- $\gamma$, anti-type II collagen autoantibodies, rheumatoid factor, and promoted an increase in beneficial microbes in the gut, including Bifidobacterium and Lactobacillus. The main findings of Sato et al. study were the beneficial effects of the Lactobacillus jensenii TL2937 in the experimental colitis induced by dextran sodium sulfate. Upon treatment with this Lactobacillus strain, mice mice presented reduced disease and myeloperoxidase activity, decreased pro-inflammatory cytokines, and increased regulatory IL-10 and IL-27 production in the intestine.

In allergic diseases, Yazdi et al. examined the impact of probiotic (Lactobacillus rhamnosus GG) administration alone or in combination with a prebiotic (turmeric powder) in asthma induced by house dust mite in BALB/c mice. The synbiotic administration decreased the number of eosinophils in bronchoalveolar lavage fluid, the Th2 lymphocytes, IL-5 and IL-13 levels, serum IgE concentrations, and hyper responsiveness to methacholine.

Lastly, to investigate the effect of obesity and intestinal dysbiosis in sepsis, Panpetch et al. orally administered $(1 \rightarrow 3)$ $\beta$-D-glucan (Candida albicans) in mice fed with high-fat diet, and then induced sepsis by cecal ligation and puncture. In the obese mice, which previously received $\beta$-D-glucan from Candida albicans, sepsis was more severe, with liver and kidney damage, endotoxemia, increased intestinal permeability, inflammatory cytokines, and gram-negative bacteria in the gut. Researchers also evaluated the effect of Lactobacillus rhamnosus L34 probiotic on sepsis, and observed an improvement in disease severity, with a decrease in the intestinal permeability, and an increase in antiinflammatory butyrate-producing commensals in the gut.

In addition, four reviews explored the mechanisms and the impact of probiotics in the modulation of the immune responses in human inflammatory conditions. A review by Yan and Polk focused on the impact of probiotics and postbiotics in intestinal homeostasis and epithelial barrier function, mucosal immune responses, and the gut-brain axis communications. Moreover, authors discussed the beneficial effects of probiotics or their metabolites in IBD and colorectal cancer, thus proposing personalized treatments based on the individual microbiome profiles. In another important study, Cristofori et al. explored the complex interactions among diet, microbiota, their metabolites and the immune system, reviewing key concepts about microbial-human evolution, hygiene hypothesis, microbiota resilience and dysbiosis. Furthermore, the probiotics' immune regulatory mechanisms in the intestine were discussed, with examples of their application in several diseases, including IBD, irritable bowel syndrome, celiac disease, obesity, autism, and Parkinson's disease, evidencing why the intestine can be considered "a door to the body". Marietta et al. compiled a complete and up-to-date information on the clinical trials based on bacterial monotherapies or multistrain probiotic treatments, as well as fecal microbiota transplantation (FMT) in several autoimmune diseases, offering a perspective on the implications and translation of experimental studies from animals to humans. The highlight of the review is the discussion on the possible use of FMT in the treatment of autoimmune diseases, including celiac disease, multiple sclerosis, rheumatoid arthritis, type 1 diabetes and Sjögren's syndrome. Lastly, Pecora et al. reviewed 
the role played by intestinal microbiota in celiac disease pathogenesis and the effects of probiotic supplementation in improving symptoms of these patients, drawing attention to the importance of carefully evaluating which probiotic to use, the dose and the length of treatment. In addition to these excellent reviews, De Oliveira et al. showed the beneficial effect of probiotics in respiratory infections, and discussed their possible use as an adjunctive therapy in COVID-19.

In a very interesting hypothesis-driven article, Ouyang et al. revisited the beneficial properties of Akkermansia muciniphila in supporting an anti-inflammatory milieu in the gut, preventing increased intestinal permeability, bacterial translocation and systemic inflammation. Most interestingly, the authors discussed the application of $A$. muciniphila supplementation in metabolic diseases and cancer, as well as alternatives to increase the abundance of this synbiotic microbe in our guts. Finally, researchers hypothesized that supplementation with A. muciniphila in patients living with HIV could decrease the barrier permeability, bacterial translocation, inflammation, and the risk to develop cardiovascular diseases or type 2 diabetes.

Lastly, the influence of diet on shaping the gut microbiota was also addressed in three studies; two reviews and one original research article. Basson et al. focused on the role of dietary fatty acids and high-fat diets in modulating the immune system via alterations of the gut microbiota and epithelial barrier permeability, reviewing mainly IBD studies. Kahalehili et al. evaluated the role of indole-3-carbinol (I3C), phytochemicals found in cruciferous vegetables, in modulating autoimmune diabetes through aryl hydrocarbon receptor (AhR) ligands.

\section{REFERENCES}

1. Donaldson GP, Lee SM, Mazmanian SK. Gut Biogeography of the Bacterial Microbiota. Nat Rev Microbiol (2016) 14(1):20-32. doi: 10.1038/nrmicro3552

2. Palm NW, de Zoete MR, Flavell RA. Immune-Microbiota Interactions in Health and Disease. Clin Immunol Orlando Fla (2015) 159(2):122-7. doi: 10.1016/j.clim.2015.05.014

3. De Luca F, Shoenfeld Y. The Microbiome in Autoimmune Diseases. Clin Exp Immunol (2019) 195(1):74-85. doi: 10.1111/cei.13158

4. Tamburini S, Shen N, Wu HC, Clemente JC. The Microbiome in Early Life: Implications for Health Outcomes. Nat Med (2016) 22(7):713-22. doi: $10.1038 / \mathrm{nm} .4142$

5. Lynch SV, Pedersen O. The Human Intestinal Microbiome in Health and Disease. N Engl J Med (2016) 375(24):2369-79. doi: 10.1056/NEJMra1600266

6. Fan Y, Pedersen O. Gut Microbiota in Human Metabolic Health and Disease. Nat Rev Microbiol (2021) 19(1):55-71. doi: 10.1038/s41579-020-0433-9

7. de Oliveira GLV, Leite AZ, Higuchi BS, Gonzaga MI, Mariano VS. Intestinal Dysbiosis and Probiotic Applications in Autoimmune Diseases. Immunology (2017) 152(1):1-12. doi: 10.1111/imm.12765

8. Marietta E, Horwath I, Balakrishnan B, Taneja V. Role of the Intestinal Microbiome in Autoimmune Diseases and its Use in Treatments. Cell Immunol (2019) 339:50-8. doi: 10.1016/j.cellimm.2018.10.005

9. Jiao Y, Wu L, Huntington ND, Zhang X. Crosstalk Between Gut Microbiota and Innate Immunity and Its Implication in Autoimmune Diseases. Front Immunol (2020) 11:282. doi: 10.3389/fimmu.2020.00282

10. Brown EM, Kenny DJ, Xavier RJ. Gut Microbiota Regulation of T Cells During Inflammation and Autoimmunity. Annu Rev Immunol (2019) 37:599624. doi: 10.1146/annurev-immunol-042718-041841

11. Levy M, Kolodziejczyk AA, Thaiss CA, Elinav E. Dysbiosis and the Immune System. Nat Rev Immunol (2017) 17(4):219-32. doi: 10.1038/nri.2017.7
The metabolized I3C from the diet induced AhR activation in the small intestine, alterations in microbiota diversity, differentiation of Th17 cells in the lamina propria, along with insulitis in non-obese diabetic (NOD) mice. In a very interesting transkingdom network analysis, to predict host-microbiota interactions, several Firmicutes phyla members were negatively regulated by I3C. Furthermore, by utilizing AhR knockout mice, they observed that Intestinimonas is downregulated by $\mathrm{AhR}$ activation. In addition to these works, Raftery et al. discussed the role of microbial-metabolized dietary metabolites, showing that the intake of saturated fats induces an increased intestinal permeability and inflammatory response. On the other hand, high soluble fiber diet, rich in vegetables and fruits, is able to suppress inflammatory mediators via SCFAs (mainly butyrate), thus inducing regulatory mechanisms and protective effects in IBD or COPD.

Collectively, the articles from this Research Topic showed important aspects of the role played by intestinal dysbiosis in inflammatory diseases. Furthermore, the strategic approaches for the use of commensals or microbiota modulators, and consequently their involvement in immune regulation were discussed and contributed to increase the knowledge in the field.

\section{AUTHOR CONTRIBUTIONS}

GO wrote the initial draft of the editorial. AF, CC, and VT revised and approved the manuscript. All authors contributed to the article and approved the submitted version.
12. Yi L, Dong X, Grenier D, Wang K, Wang Y. Research Progress of Bacterial Quorum Sensing Receptors: Classification, Structure, Function and Characteristics. Sci Total Environ (2021) 763:143031. doi: 10.1016/j.scitotenv.2020.143031

13. Deng Z, Luo XM, Liu J, Wang H. Quorum Sensing, Biofilm, and Intestinal Mucosal Barrier: Involvement the Role of Probiotic. Front Cell Infect Microbiol (2020) 10:538077. doi: 10.3389/fcimb.2020.538077

14. Ratajczak W, Rył A, Mizerski A, Walczakiewicz K, Sipak O, Laszczyńska M. Immunomodulatory Potential of Gut Microbiome-Derived Short-Chain Fatty Acids (SCFAs). Acta Biochim Pol (2019) 66(1):1-12. doi: 10.18388/abp.2018_2648

15. Smith PM, Howitt MR, Panikov N, Michaud M, Gallini CA, Bohlooly-Y M, et al. The Microbial Metabolites, Short-Chain Fatty Acids, Regulate Colonic Treg Cell Homeostasis. Science (2013) 341(6145):569-73. doi: 10.1126/ science. 1241165

16. de Souza PR, Guimarães FR, Sales-Campos H, Bonfá G, Nardini V, Chica JEL, et al. Absence of NOD2 Receptor Predisposes to Intestinal Inflammation by a Deregulation in the Immune Response in Hosts that are Unable to Control Gut Dysbiosis. Immunobiology (2018) 223(10):577-85. doi: 10.1016/ j.imbio.2018.07.003

17. Hu Y, Peng J, Li F, Wong FS, Wen L. Evaluation of Different Mucosal Microbiota Leads to Gut Microbiota-Based Prediction of Type 1 Diabetes in NOD Mice. Sci Rep (2018) 8(1):15451. doi: 10.1038/s41598-018-33571-z

18. Simon M-C, Reinbeck AL, Wessel C, Heindirk J, Jelenik T, Kaul K, et al. Distinct Alterations of Gut Morphology and Microbiota Characterize Accelerated Diabetes Onset in Nonobese Diabetic Mice. J Biol Chem (2020) 295(4):969-80. doi: 10.1016/S0021-9258(17)49908-X

19. Neuman V, Cinek O, Funda DP, Hudcovic T, Golias J, Kramna L, et al. Human Gut Microbiota Transferred to Germ-Free NOD Mice Modulate the Progression Towards Type 1 Diabetes Regardless of the Pace of Beta Cell Function Loss in the Donor. Diabetologia (2019) 62(7):1291-6. doi: 10.1007/ s00125-019-4869-2 
20. Moshkelgosha S, Masetti G, Berchner-Pfannschmidt U, Verhasselt HL, Horstmann M, Diaz-Cano S, et al. Gut Microbiome in BALB/c and C57BL/ 6J Mice Undergoing Experimental Thyroid Autoimmunity Associate With Differences in Immunological Responses and Thyroid Function. Horm Metab Res Horm Stoffwechselforschung Horm Metab (2018) 50(12):932-41. doi: 10.1055/a-0653-3766

21. Masetti G, Moshkelgosha S, Köhling H-L, Covelli D, Banga JP, BerchnerPfannschmidt U, et al. Gut Microbiota in Experimental Murine Model of Graves' Orbitopathy Established in Different Environments May Modulate Clinical Presentation of Disease. Microbiome (2018) 6(1):97. doi: 10.1186/ s40168-018-0478-4

22. Maeda Y, Kurakawa T, Umemoto E, Motooka D, Ito Y, Gotoh K, et al. Dysbiosis Contributes to Arthritis Development via Activation of Autoreactive T Cells in the Intestine. Arthritis Rheumatol Hoboken NJ (2016) 68(11):2646-61. doi: 10.1002/art.39783

23. Chen J, Wright K, Davis JM, Jeraldo P, Marietta EV, Murray J, et al. An Expansion of Rare Lineage Intestinal Microbes Characterizes Rheumatoid Arthritis. Genome Med (2016) 8(1):43. doi: 10.1186/s13073-016-0299-7

24. Jubair WK, Hendrickson JD, Severs EL, Schulz HM, Adhikari S, Ir D, et al. Modulation of Inflammatory Arthritis in Mice by Gut Microbiota Through Mucosal Inflammation and Autoantibody Generation. Arthritis Rheumatol Hoboken NJ (2018) 70(8):1220-33. doi: 10.1002/art.40490

25. Johnson BM, Gaudreau M-C, Gudi R, Brown R, Gilkeson G, Vasu C. Gut Microbiota Differently Contributes to Intestinal Immune Phenotype and Systemic Autoimmune Progression in Female and Male Lupus-Prone Mice. J Autoimmun (2020) 108:102420. doi: 10.1016/j.jaut.2020.102420

26. Ma Y, XuX, Li M, Cai J, Wei Q, Niu H. Gut Microbiota Promote the Inflammatory Response in the Pathogenesis of Systemic Lupus Erythematosus. Mol Med Camb Mass (2019) 25(1):35. doi: 10.1186/s10020-019-0102-5

27. Luo XM, Edwards MR, Mu Q, Yu Y, Vieson MD, Reilly CM, et al. Gut Microbiota in Human Systemic Lupus Erythematosus and a Mouse Model of Lupus. Appl Environ Microbiol (2018) 84(4):e02288-17. doi: 10.1128/ AEM.02288-17

28. de Paiva CS, Jones DB, Stern ME, Bian F, Moore QL, Corbiere S, et al. Altered Mucosal Microbiome Diversity and Disease Severity in Sjögren Syndrome. Sci Rep (2016) 6:23561. doi: 10.1038/srep23561

29. Wu H-J, Wu E. The Role of Gut Microbiota in Immune Homeostasis and Autoimmunity. Gut Microbes (2012) 3(1):4-14. doi: 10.4161/gmic.19320

30. Rosser EC, Mauri C. A Clinical Update on the Significance of the Gut Microbiota in Systemic Autoimmunity. J Autoimmun (2016) 74:85-93. doi: 10.1016/j.jaut.2016.06.009
31. Ruff WE, Greiling TM, Kriegel MA. Host-Microbiota Interactions in Immune-Mediated Diseases. Nat Rev Microbiol (2020) 18(9):521-38. doi: 10.1038/s41579-020-0367-2

32. Mu Q, Kirby J, Reilly CM, Luo XM. Leaky Gut as a Danger Signal for Autoimmune Diseases. Front Immunol (2017) 8:598. doi: 10.3389/ fimmu.2017.00598

33. Lerner A, Aminov R, Matthias T. Dysbiosis May Trigger Autoimmune Diseases via Inappropriate Post-Translational Modification of Host Proteins. Front Microbiol (2016) 7:84. doi: 10.3389/fmicb.2016.00084

34. Lee J, Im JP, Han K, Park S, Soh H, Choi K, et al. Risk of Inflammatory Bowel Disease in Patients With Chronic Obstructive Pulmonary Disease: A Nationwide, Population-Based Study. World J Gastroenterol (2019) 25 (42):6354-64. doi: 10.3748/wjg.v25.i42.6354

35. Labarca G, Drake L, Horta G, Jantz MA, Mehta HJ, Fernandez-Bussy S, et al. Association Between Inflammatory Bowel Disease and Chronic Obstructive Pulmonary Disease: a Systematic Review and Meta-Analysis. BMC Pulm Med (2019) 19(1):186. doi: 10.1186/s12890-019-0963-y

36. Vutcovici M, Bitton A, Ernst P, Kezouh A, Suissa S, Brassard P. Inflammatory Bowel Disease and Risk of Mortality in COPD. Eur Respir J (2016) 47 (5):1357-64. doi: 10.1183/13993003.01945-2015

Conflict of Interest: AF holds stocks in Alba Therapeutics and receives financial support from Takeda Pharmaceuticals. VT has received financial support from Elysium Health and Evelo Biosciences.

The remaining authors declare that the research was conducted in the absence of any commercial or financial relationships that could be construed as a potential conflict of interest.

Publisher's Note: All claims expressed in this article are solely those of the authors and do not necessarily represent those of their affiliated organizations, or those of the publisher, the editors and the reviewers. Any product that may be evaluated in this article, or claim that may be made by its manufacturer, is not guaranteed or endorsed by the publisher.

Copyright $\odot 2021$ de Oliveira, Cardoso, Taneja and Fasano. This is an open-access article distributed under the terms of the Creative Commons Attribution License (CC BY). The use, distribution or reproduction in other forums is permitted, provided the original author(s) and the copyright owner(s) are credited and that the original publication in this journal is cited, in accordance with accepted academic practice. No use, distribution or reproduction is permitted which does not comply with these terms. 